ren Präparaten eine Widerstandsänderung im Leitungsband überlagert ist, muß noch durch eingehende Temperaturanalysen untersucht werden.

Für wertvolle Diskussionsbeiträge habe ich vor allem den Herren Dr. O. G. Folberth, Dr. R. Gremmelmaier und Dr. H. Weiss zu danken. Besonderen Dank schulde ich Herrn Dr. C. Herring für die Überlassung eines Manuskriptes seiner Arbeit über den Einfluß von Inhomogenitäten auf die galvanomagnetischen Effekte.

\section{Anhang}

Herleitung der Gl. (1)

Für den im Experiment beobachteten spezifischen Widerstand $\left(\varrho_{x x}\right)$ eff parallel zur Probenlängsachse findet Herring:

$$
\begin{gathered}
\left(\varrho_{x x}\right)_{\mathrm{eff}}=n\left\langle\frac{1}{n}\right\rangle \varrho_{x x}+n\left[\left\langle\frac{1}{n}\right\rangle-\frac{1}{\langle n\rangle}\right] \\
\cdot\left[\frac{\varrho_{x y} \varrho_{y z} \varrho_{z x}+\varrho_{x z} \varrho_{z y} \varrho_{y x}-\varrho_{x y} \varrho_{y x} \varrho_{z z}-\varrho_{x z} \varrho_{z x} \varrho_{y y}}{\varrho_{y y} \varrho_{z z}-\varrho_{y z} \varrho_{z y}}\right] .
\end{gathered}
$$

Dabei bedeutet $n$ die Elektronenkonzentration einer beliebig herausgegriffenen Schicht und $\varrho_{\mu \nu}$ die $\mu \nu$-te Komponente des Widerstandstensors aus der gleichen betrachteten Schicht. Da die Beweglichkeit in allen Schichten gleich groß sein soll, ist das Produkt $n \varrho_{\mu \nu}$ für alle Schichten konstant.

Um nun Gl. (A 1) auf unser Problem bei InSb anwenden zu können, gehen wir von einer Beziehung aus, welche sich durch Hinzunahme der in $B$ linearen Glieder aus Gl. (10) der früheren Arbeit ${ }^{1}$ ergibt.

$E_{\lambda}=\varrho_{0} j_{\lambda}+R_{\mathrm{H}}(j \times B)_{\lambda}+\alpha_{12} j_{\lambda} B^{2}-\alpha_{12} B_{\lambda}(j \cdot B)$; $a_{12}$ ist der Koeffizient der transversalen Widerstandsänderung. Hieraus berechnen sich die Tensorkomponenten in folgender Weise:

$$
\begin{aligned}
& \varrho_{x x}=\varrho_{0}+\alpha_{12}\left(B^{2}-B_{x}^{2}\right), \\
& \varrho_{y x}=-R_{\mathrm{H}} B_{z}-\alpha_{12} B_{x} B_{y}, \\
& \varrho_{z x}=R_{\mathrm{H}} B_{y}-\alpha_{12} B_{x} B_{z}, \\
& \varrho_{x y}=R_{\mathrm{H}} B_{z}-\alpha_{12} B_{x} B_{y}, \quad \varrho_{x z}=-R_{\mathrm{H}} B_{y}-\alpha_{12} B_{x} B_{z}, \\
& \varrho_{y y}=\varrho_{0}+\alpha_{12}\left(B^{2}-B_{y}{ }^{2}\right), \quad \varrho_{y z}=R_{\mathrm{H}} B_{x}-\alpha_{12} B_{y} B_{z}, \\
& \varrho_{z y}=-R_{\mathrm{H}} B_{x}-\alpha_{12} B_{y} B_{z}, \varrho_{z z}=\varrho_{0}+\alpha_{12}\left(B^{2}-B_{z}{ }^{2}\right) .
\end{aligned}
$$

Nehmen wir nun an

1. $B$ liege in der $x-z$-Ebene,

2. $\varphi$ sei der Winkel zwischen $B$ und der $x$-Achse, also $B_{x}=B \cos \varphi, B_{z}=B \sin \varphi$,

3. eine physikalische Widerstandsänderung sei nicht vorhanden, d. h. $\alpha_{12}=0$ (dies kann zumindest für starke Dotierung aus der unteren ausgezogenen Kurve in Abb. 4 der früheren Arbeit ${ }^{1}$ geschlossen werden), so folgt durch Einsetzen der Tensorkomponenten aus (A 3) in (A 1) unmittelbar

$\left(\varrho_{x x}\right)_{\mathrm{eff}}=n\left\langle\frac{1}{n}\right\rangle \varrho_{0}+n\left[\left\langle\frac{1}{n}\right\rangle-\frac{1}{\langle n\rangle}\right] \varrho_{0} \frac{(\mu B)^{2} \sin ^{2} \varphi}{1+(\mu B)^{2} \cos ^{2} \varphi}$.

Daraus erhält man für die relative Änderung des Widerstandes im Magnetfeld in Abhängigkeit vom Drehwinkel

$$
\begin{array}{r}
\frac{\Delta R}{R_{0}}=\frac{\left(\varrho^{(B)} x x\right) \text { eff }-\left(\varrho^{(0)} x x\right)_{\text {eff }}}{\left(\varrho^{(0)} x x\right) \text { eff }} \\
=\left[1-\frac{1}{\langle n\rangle\langle 1 / n\rangle}\right] \frac{(\mu B)^{2} \sin ^{2} \varphi}{1+(\mu B)^{2} \cos ^{2} \varphi} .
\end{array}
$$

\title{
Der Einfluß der elektronischen Polarisation auf Exzitonen in Alkalihalogenidkristallen
}

\author{
Von Wolfgang Weller \\ Aus dem Institut für theoretische Physik der Karl-Marx-Universität Leipzig \\ (Z. Naturforschg. 16 a, 401-410 [1961] ; eingegangen am 28. November 1960)
}

\begin{abstract}
Bei Exzitonen in Alkalihalogeniden ist die Kontinuumsnäherung (Methode der effektiven Masse) nicht anwendbar, da der mittlere Abstand von Elektron und Loch kleiner als der Abstand benachbarter Halogenionen ist. Mit dem von Toyozawa und Haken und Schotтky entwickelten Modell wird der Einfluß der elektronischen Polarisation auf das Exziton unter Berücksichtigung der Gitterstruktur des Kristalls berechnet. Die elektronische Polarisation ist zu vernachlässigen, wenn sich das angeregte Elektron und das Loch am gleichen Halogenion befinden. Bei größeren Abständen von Elektron und Loch muß im Cоuцомв-Gesetz der volle Wert der optischen Dielektrizitätskonstante (DK) benutzt werden. Die Polarisationsenergie für sehr große Abstände wird berechnet, für NaCl ergibt sich $-2,8 \mathrm{eV}$. Das Modell von Toyozawa und Haken und Sснотткy wird schließlich so erweitert, daß es die Lorentz-Korrektur für die optische DK liefert.
\end{abstract}

Zur Behandlung der elektronischen Polarisation in Kristallen wurde von Toyozawa ${ }^{1}$ und Haken und Sсноттку ${ }^{2}$ ein Modell entwickelt. Die elektronische Polarisation entsteht dabei durch Anregung von Valenzelektronen, die sich an Ionen oder Atomen befin- den, die den polarisierenden Ladungen benachbart sind.

1 Y. Toyozawa, Progr. Theor. Phys. 12, 421 [1954].

2 H. Haken u. W. Schottky, Z. phys. Chem. N. F. 16, 218 [1958]. 
Betrachten wir ein Exziton, so wirken sowohl das angeregte Elektron wie auch das Loch im Valenzband polarisierend auf den Kristall. HaKen und Sсноттку $^{2}$ berechneten die elektronische Polarisation für Exzitonen mit der Kontinuumsnäherung, d. h. für den Fall, daß der Radius des wasserstoffähnlichen Exzitons groß gegen die Gitterkonstante ist. Sie erhalten für das durch die elektronische Polarisation abgeänderte Potential zwischen angeregtem Elektron und Loch

$$
-\frac{e^{2}}{r}+\left(1-\frac{1}{\varepsilon_{\text {opt }}}\right) \frac{e^{2}}{r}\left[1-\frac{e^{-u_{1} r}+e^{-u_{2} r}}{2}\right]+\text { const }
$$

$r$ ist der Abstand Elektron-Loch; $u_{i}=\sqrt{2 m_{i} \Delta E / \hbar^{2}}$; $m_{1}, m_{2}$ sind die effektiven Massen von Elektron und Loch an den Bandrändern; $\Delta E$ ist die Anregungsenergie eines Valenzelektrons; $\varepsilon_{\text {opt }}$ ist die optische DK, die sich aus dem Modell ergibt.

Das Potential (1) rechtfertigt für große Abstände die Verwendung der DK im Coulomb-Gesetz und vermittelt für kleine Abstände den Übergang zum Coulomb-Gesetz $e^{2} / r$ ohne DK.

Für den Grundzustand des Exzitons in Alkalihalogeniden ist die Kontinuumsnäherung (W ANNIERsche Methode der effektiven Masse) nicht anwendbar, da der mittlere Abstand von Elektron und Loch kleiner als der Abstand $n_{0}$ benachbarter Halogenionen ist ${ }^{3}$. $\left(n_{0}=7,5 a_{0}\right.$ für $\mathrm{NaCl}, a_{0}$ ist der erste Bohrsche Radius.) Von Dexter ${ }^{4}$ und Muto und Okuno ${ }^{5}$ wurde das Exziton in $\mathrm{NaCl}$ und anderen Alkalihalogeniden mit Hilfe der Methode der effektiven Masse behandelt. Das Ergebnis für den mittleren Abstand von Elektron und Loch $\left(\approx 3 a_{0}\right.$ für $\mathrm{NaCl}$ ) beweist die Nichtanwendbarkeit dieser Methode.

Für Alkalihalogenide ist die Anregungsenergie $\Delta E$ der Valenzelektronen gro ${ }^{6}$, die $u_{i}{ }^{-1}$ sind dann klein. Für $\mathrm{NaCl}$ ergeben sich für die $u_{i}{ }^{-1}$ Längen der Größe $1 a_{0}$. Die elektronische Polarisation bildet sich deshalb (vermutlich) auch in dem Gebiet aus, in dem die zur Gewinnung von Gl. (1) benutzte Kontinuumsnäherung versagt.

Wir behandeln in dieser Arbeit die Wechselwirkung (WW) des Exzitons mit der elektronischen Polarisation ohne Kontinuumsnäherung. Es ergeben sich u. a. die Werte für die DK, die in das Coulomb-

3 Vgl. R. A. Pappert, Phys. Rev. 119, 525 [1960].

4 D. L. Dexter, Phys. Rev. 83, 435 [1951] ; 108, 707 [1957].

5 T. Muto u. H. Okuno, J. Phys. Soc., Japan 11, 633 [1956]; 12, 108 [1957]. - T. Muto, S. Oуama u. H. Okuno, Progr. Theor. Phys. 20, 804 [1958].
Gesetz beim Exzitonmodell einzusetzen sind. Muto und Окимо ${ }^{5}$ verwenden stets den vollen Wert der optischen DK.

Im 1. Abschnitt benutzen wir die Darstellung von SLATER $^{7}$ für das Exziton und behandeln die WW des Exzitons mit der elektronischen Polarisation mit dem Modell von Haken und Schotткy ${ }^{2}$. Im 2. Abschnitt berechnen wir das durch die elektronische Polarisation erzeugte Potential zwischen Elektron und Loch unter Berücksichtigung der Gitterstruktur des Kristalls. Im 3. Abschnitt berechnen wir den vom Abstand unabhängigen Anteil der Polarisationsenergie, der die Polarisationsenergie zweier sehr weit entfernter Ladungen darstellt. Dieser Anteil ist für den Bandabstand des Kristalls und die Energieberechnung des Exzitons wichtig. Im 4. Abschnitt wird das Modell für die WW des Exzitons mit der elektronischen Polarisation so erweitert, daß sich für die optische DK die Lorentz-Korrektur ergibt.

Die Rechnungen sind auf alle Alkalihalogenide (mit kubisch flächenzentriertem Gitter) anwendbar; spezielle Zahlenwerte und Abschätzungen werden immer für $\mathrm{NaCl}$ angegeben.

Die Ionenpolarisation kann wegen des kleinen Exzitonradius in Alkalihalogeniden für den Grundzustand außer acht bleiben; sie trägt jedoch zum abstandsunabhängigen Anteil der Polarisationsenergie bei (vgl. Abschnitt 3) .

\section{Modell für Exziton und elektronische Polarisation}

Wir behandeln die elektronische Polarisation im Anschluß an Haken und Sсноттку ${ }^{2}$. Für das Exziton benutzen wir die Darstellung von SLATER ${ }^{7}$, die auch für Exzitonen mit kleinem Elektron-Loch-Abstand geeignet ist.

Der Hamilton-Operator der Valenzelektronen $\left(\mathrm{Cl}^{-}\right.$-3p-Elektronen für $\left.\mathrm{NaCl}\right)$ lautet

$$
H=\sum_{i} H_{0}\left(\mathrm{r}_{i}\right)+\frac{1}{2} \sum_{i \neq j} \frac{e^{2}}{\left|\mathrm{r}_{i}-\mathrm{r}_{j}\right|} .
$$

$H_{0}(\mathrm{r})$ ist der Hamilton-Operator eines Elektrons im Felde der Alkaliionen und der Halogenionen mit fortgenommenen p-Elektronen. Der zweite Term ist die Coulomb-Energie der Valenzelektronen. Die

\footnotetext{
6 Siehe Anhang 1.

7 J. C. Slater, Technical Report No. 6, Massachusetts Institute of Technology, 1954.
} 
W AnNiER-Funktionen des Valenzbandes seien

$$
a_{\mu}(\mathfrak{r}-\mathfrak{n}) \quad(\mu=1,2,3),
$$

die W ANnier-Funktionen des Leitungsbandes

$$
a(\mathfrak{r}-\mathfrak{n}) ;
$$

$\mathfrak{n}$ bezeichnet den Halogengitterplatz. Zur Vereinfachung sehen wir hier vom Spin der Elektronen ab.

Die Wellenfunktion für den Grundzustand des Kristalls lautet

$$
\begin{array}{c|ccc} 
& \ldots a_{1}\left(\mathrm{r}_{1}-\mathfrak{n}\right) & a_{2}\left(\mathrm{r}_{1}-\mathfrak{n}\right) & \ldots a_{1}\left(\mathrm{r}_{1}-\mathfrak{n}\right) \ldots \\
\frac{1}{V(3 N) !} & \ldots a_{1}\left(\mathrm{r}_{2}-\mathfrak{n}\right) & \ldots & \ldots
\end{array} \mid
$$

$N$ ist die Zahl der Halogenionen. - Für die Wellenfunktion des Exzitons ohne elektronische Polarisation schreiben wir

$$
\left.|\mathfrak{R}\rangle=\sum_{\mathfrak{n}} U(\mathfrak{n}) \underset{\mathfrak{n}}{\stackrel{\mathfrak{R}}{ }}\right\rangle
$$

$$
\left.=\sum_{\mathrm{n}} U(\mathfrak{n}) \frac{1}{\sqrt{ } N} \sum_{\mathrm{m}} e^{i \boldsymbol{\Omega}_{\mathrm{m}}{ }_{\mathrm{m}+\mathfrak{m}}^{\mathfrak{m}}}\right\rangle .
$$

$\Re$ ist der Impuls des Exzitons. $\mid \begin{aligned} & \mathfrak{m} \\ & \mathfrak{m}+\mathfrak{n}\end{aligned}>$ entsteht aus der Slater-Determinante (3), indem die Spalte $a_{\mu}\left(\mathfrak{r}_{i}-\mathfrak{m}\right)$ durch die Spalte $a\left(\mathfrak{r}_{i}-\mathfrak{m}-\mathfrak{n}\right)$ ersetzt wird. Die Orientierung $\mu$ des Exzitons ist in (4) nicht explizit angedeutet worden, sie bleibt im Verlauf der Rechnung unverändert. $U(\mathfrak{n})$ ist die Verteilungsfunktion des angeregten Elektrons, das sich um das Loch gruppiert.

Wir führen mit Haken und Sснотткy zur Beschreibung der elektronischen Polarisation Erzeugungs- und Vernichtungsoperatoren für Paarzustände ein. $\beta_{\mathfrak{n} v}^{+}$ersetzt in einer SLATER-Determinante die Spalte $a_{v}\left(\mathrm{r}_{i}-\mathfrak{n}\right)$ durch $a\left(\mathrm{r}_{i}-\mathfrak{n}\right)$, erzeugt einen Paarzustand; $\beta_{n v}$ vernichtet einen Paarzustand ${ }^{8}$. Die elektronische Polarisation wird durch die Beimengung solcher Paarzustände erzeugt. Wegen der Translationssymmetrie des Kristalls erfolgt der Übergang zu laufenden Wellen

$$
\beta_{\mathfrak{t} v}^{+}=\frac{1}{\sqrt{N}} \sum_{\mathrm{n}} e^{i \neq n} \beta_{\mathrm{n} v}^{+} .
$$

Wir sehen von Überlappungstermen ab, da die Zahl der angeregten Paarzustände $\ll N$, und betrachten $\operatorname{die} \beta_{\mathfrak{t} v}^{+}$und $\beta_{\mathfrak{t} z}$ als Bose-Operatoren.

\footnotetext{
${ }_{8} \beta_{\mathfrak{n} v}^{+}$ergibt Null, wenn die betreffende Spalte schon durch Funktionen des Leitungsbandes ersetzt ist; analog ergibt

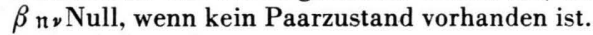

9 Siehe Anhang 1.
}

Für das Exziton mit elektronischer Polarisation benutzen wir als Basisfunktionen die Funktionen $\left|\begin{array}{l}\Re \\ \pi\end{array}\right\rangle$ und die daraus durch Anwendung der $\beta_{\mathfrak{f} v}^{+}$ entstehenden normierten Funktionen. Den HamiltonOperator $H$ schreiben wir in der Form

$$
H=H_{\mathrm{E}}+\sum_{\mathfrak{f} v} \Delta E(\mathfrak{f} v) \beta_{\mathfrak{f} v}^{+} \beta_{\mathfrak{f} v}+H_{\mathrm{WW}} .
$$

$H_{\mathrm{E}}$ hat nur Matrixelemente, die vom Exzitonanteil herrühren. $\Delta E(\mathfrak{f} v)$ ist die Energie eines Paarzustandes ${ }^{9}$. Die Orientierungen der Paarzustände wählen wir so, daß $p_{1} \| \mathfrak{f}$ für $v=1$ (longitudinale Paarzustände) und $\mathfrak{p}_{2,3} \perp \mathfrak{f}$ für $v=2,3$ (transversale Paarzustände). Dabei sind die Dipolmomente

$$
\mathfrak{p}_{v}=e \int a^{*}(\mathrm{r}) \mathrm{r} a_{v}(\mathrm{r}) \mathrm{d} \tau,
$$

die wir als reell betrachten. Bei dieser Wahl der Orientierungen sind alle Paarzustände voneinander entkoppelt ${ }^{2}$.

$H_{\mathrm{Ww}}$ hat Matrixelemente, die in der Anzahl der Paarzustände nicht diagonal sind, und beschreibt die WW des Exzitons mit den Paarzuständen. Wir schreiben $H_{\mathrm{WW}}$ in der Form

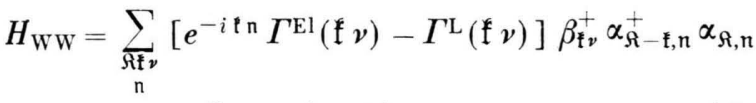

$$
\begin{aligned}
& + \text { (herm. konj.). }
\end{aligned}
$$

Die Operatoren $\alpha$ sorgen für die Erhaltung des Impulses; $\alpha_{\Omega, n}$ vernichtet den Exzitonanteil mit Impuls $\Re$ und Elektron-Loch-Abstand $\mathfrak{n}$ und $\alpha_{\Omega}^{+}-\mathfrak{k}, \mathfrak{n}$ erzeugt diesen Anteil mit geändertem Impuls $\Re-\mathfrak{f}$ wieder. Die $\Gamma$ sind Matrixelemente der CoulombEnergie mit unseren Basisfunktionen

$$
\begin{aligned}
& \Gamma^{\mathrm{El}}(\mathfrak{f} v)=\frac{1}{\sqrt{N}} \sum_{\mathrm{m}} e^{i \ell \mathrm{m}} \\
& \cdot \int a^{*}\left(\mathrm{r}_{1}\right) a_{v}\left(\mathrm{r}_{1}\right) \frac{e^{2}}{\left|\mathrm{r}_{1}-\mathrm{r}_{2}\right|}\left|a\left(\mathrm{r}_{2}-\mathrm{m}\right)\right|^{2} \mathrm{~d} \tau_{12}, \\
& \Gamma^{\mathrm{L}}(\mathfrak{f} \nu)=\frac{1}{\sqrt{N}} \sum_{\mathrm{m}} e^{i \mathfrak{m} \mathrm{m}} \\
& \text { - } \int a^{*}\left(\mathrm{r}_{1}\right) a_{v}\left(\mathrm{r}_{1}\right) \frac{e^{2}}{\left|\mathrm{r}_{1}-\mathrm{r}_{2}\right|}\left|a_{\mu}\left(\mathrm{r}_{2}-\mathrm{m}\right)\right|^{2} \mathrm{~d} \tau_{12} .
\end{aligned}
$$

$\Gamma^{\mathrm{El}}$ beschreibt die $\mathrm{WW}$ des Elektrons, $\Gamma^{\mathrm{L}}$ die des Loches mit den Paarzuständen. Entwicklung von $1 /\left|r_{1}-r_{2}\right|$ nach $r_{1}$ und $r_{2}-m$ liefert in der ersten nicht verschwindenden Näherung (Pol-Dipol-Näherung von Haken und Sснотткy) für die $\Gamma$

$$
\Gamma^{\mathrm{El}}(\mathfrak{f} v)=\frac{e}{V N} \sum_{\mathfrak{m} \neq 0} e^{i \mathfrak{t} \mathfrak{m}} \frac{\mathfrak{m} \mathfrak{p}_{v}}{|\mathfrak{m}|^{3}}=\Gamma^{\mathrm{L}}(\mathfrak{f} v) .
$$

In dieser Näherung, in der die verschiedene Ausdehnung der $W_{\text {ANNIER-Funktionen vom Elektron und }}$ 
vom Loch vernachlässigt wird, ergibt der Exzitonanteil $\left|\begin{array}{l}\Re \\ 0\end{array}\right\rangle$, bei dem sich Elektron und Loch am gleichen Halogenion befinden, keine $\mathrm{WW}$ mit den Paarzuständen.

In der nächsten Näherung ergibt sich

$$
\begin{aligned}
\Gamma^{\mathrm{El}}(\mathfrak{k} v)-\Gamma^{\mathrm{L}}(\mathfrak{k} v)= & \frac{15}{2} \frac{e}{V N} \sum_{\mathfrak{m} \neq 0} e^{i \mathfrak{l} \mathrm{m}} \frac{\mathfrak{m} \mathfrak{p}_{v}}{|\mathfrak{m}|^{7}} \\
& \times\left\{(\mathfrak{r}, \mathfrak{m}) \underset{\mathrm{El}}{2}-(\mathrm{r}, \mathfrak{m})_{\mathrm{L}}^{2}\right\}
\end{aligned}
$$

mit $(\mathfrak{r}, \mathfrak{m}) \underset{\mathrm{El}}{2}=\int|a(\mathfrak{r})|^{2}(\mathrm{r}, \mathfrak{m})^{2} \mathrm{~d} \tau$ und $(\mathfrak{r}, \mathfrak{m}){ }_{\mathrm{L}}^{2}$ analog. Die verschiedene Ausdehnung der W AnNIER-Funktionen ergibt dann auch für den Exzitonanteil $\left|\begin{array}{l}\mathcal{R} \\ 0\end{array}\right\rangle$ eine geringe $\mathrm{WW}$ mit den Paarzuständen.

Wir verwandeln die Gittersummen in (9) in ein Integral über das Volumen des Kristalls. Die Einschränkung $\mathfrak{m} \neq 0$ wird dadurch berücksichtigt, daß bei der Integration eine Kugel um den Ursprung mit Radius $R_{0}$ ausgelassen wird. $4 \pi R_{0}{ }^{3} / 3=\sigma^{-1}$, $\sigma$ ist die Zahl der Halogenionen pro Volumeneinheit. (Für $\mathrm{NaCl}$ ist $R_{0}=4,2 a_{0}$.) Die elementare Integration ergibt

$$
\Gamma^{\mathrm{El}}(\mathfrak{f} \|)=4 \pi i e \frac{\sigma p}{\sqrt{ } N k} \frac{\sin k R_{0}}{k R_{0}} .
$$

Bei dieser Integralapproximation tritt nur eine WW mit den longitudinalen Paarzuständen auf. (Für $v=1$ wird die Bezeichnung $\|$ verwendet; $p=\left|p_{\|}\right|$.) HaKen und SchotтKy benutzen für die $\Gamma$

$$
\Gamma^{\mathrm{El}}(\mathfrak{f} \|) \approx 4 \text { лi i } \sigma p / \sqrt{N} k .
$$

Gl. (11) geht für $k R_{0} \ll 1$ in Gl. (12) über.

In unserem WW-Operator (7) wurde nur die Absorption und die Emission je eines Paarzustandes berücksichtigt. Die Cоuцомв-Energie hat jedoch auch Matrixelemente, die zur simultanen Absorption und Emission zweier Paarzustände gehören. Wir kommen darauf in Abschn. 4 zurück.

\section{Berechnung des WW-Potentials}

Für die Wellenfunktion des Exzitons mit elektronischer Polarisation setzen wir an

$$
\begin{aligned}
|\rangle= & \sum_{\mathfrak{n}} U(\mathfrak{n}) \\
& \cdot\left[c_{0}(\mathfrak{n})\left|\begin{array}{l}
\mathfrak{R} \\
\mathfrak{n}
\end{array}\right\rangle+\sum_{\mathfrak{i} v} c_{\mathfrak{f} v}(\mathfrak{n}) \beta_{\mathfrak{f} v}^{+}\left|\begin{array}{l}
\mathfrak{\Omega}-\mathfrak{f} \\
\mathfrak{n}
\end{array}\right\rangle+\ldots\right] .
\end{aligned}
$$

Zur Berechnung der Energie und der Wellenfunk-

${ }^{10}$ Siehe Anhang 1. tion des Grundzustandes ist dann das Funktional $\bar{H}=\langle|H|\rangle$ durch geeignete Wahl der $U$ und $c$ zu einem Minimum zu machen. Wir bestimmen das Minimum bezüglich der $c$. In dem verbleibenden, nur noch von $U$ abhängigen Funktional erscheint dann die WW des Exzitons mit den Paarzuständen als WW-Potential zwischen Elektron und Loch.

Die WW des Exzitons mit den Paarzuständen ist von ähnlichem Typ wie die WW mit den optischen Ionenschwingungen. Für weit ausgedehnte Exzitonen lassen sich von den Ionenschwingungen her bekannte Methoden verwenden ${ }^{2}$.

Wir werden zur Berechnung des WW-Potentials den Rückstoß auf das Exziton, der bei Emission oder Absorption von Paarzuständen entsteht, vernachlässigen. Die Rückstoßenergie ist klein gegen die Anregungsenergie $\Delta E$ eines Paarzustandes ${ }^{10}$; für $\mathrm{NaCl}$ ist $\Delta E \approx 13 \mathrm{eV}$. Unsere Methode ist für den Fall der WW mit den Ionenschwingungen nicht anwendbar. Die Rolle von $\Delta E$ spielt dort die Phononenergie $\hbar \omega(\approx 0,03 \mathrm{eV}$ für $\mathrm{NaCl})$, so daß dann die Rückstoßenergie den wesentlichen Anteil bildet.

Es ist nützlich, noch die folgenden Wellenfunktionen (ohne Bezug auf Slater-Determinanten) im Raum der Bose-Operatoren $\beta$ einzuführen

$$
\mid \mathfrak{n})=c_{0}(\mathfrak{n}) \Phi_{0}+\sum_{\mathfrak{i} v} c_{\mathfrak{f} v}(\mathfrak{n}) \beta_{\mathfrak{f} v}^{+} \Phi_{0}+\ldots,
$$

$\Phi_{0}$ bedeutet, daß kein Paarzustand angeregt ist. Für $\bar{H}$ ergibt sich

$$
\begin{aligned}
\bar{H}= & \langle|H|\rangle \\
= & \sum_{\mathfrak{n} \mathfrak{n}} U^{*}(\mathfrak{n}) U(\overline{\mathfrak{l}})\left\langle\begin{array}{c}
\mathfrak{R} \\
\mathfrak{n}
\end{array}\left|H_{E}\right| \begin{array}{l}
\mathfrak{R} \\
\mathfrak{n}
\end{array}\right\rangle(\mathfrak{n} \mid \overline{\mathfrak{n}}) \\
& +\sum_{\mathfrak{n}}|U(\mathfrak{n})|^{2}\left(\mathfrak{n}\left|H_{\mathrm{P}}(\mathfrak{n})\right| \mathfrak{n}\right) \\
H_{\mathrm{P}}(\mathfrak{n})= & \sum_{\mathfrak{l} v}\left(e^{-i \mathfrak{l} \mathfrak{n}} \Gamma^{\mathrm{El}}(\mathfrak{f} v)-\Gamma^{\mathrm{L}}(\mathfrak{f} v)\right) \beta_{\mathfrak{f} v}^{+} \\
& +\sum_{\mathfrak{f} v} \Delta E(\mathfrak{f} v) \beta_{\mathfrak{t} v}^{+} \beta_{\mathfrak{f} v} .
\end{aligned}
$$
+ (herm. konj.)

Dabei wurde im ersten Term von (15) bei den Anteilen, die angeregte Paarzustände enthalten, die erwähnte Vernachlässigung des Rückstoßes vorgenommen. Wir eliminieren mit Hilfe einer kanonischen Transformation die in den $\beta$ linearen Terme von $H_{\mathrm{P}}$.

$$
\begin{aligned}
S_{\mathfrak{n}}^{-1} \beta_{\mathfrak{t} v} S_{\mathfrak{n}} & =\beta_{\mathfrak{t} v}+f_{\mathfrak{t} v}(\mathfrak{n})\left(\mathrm{s} . \text { Anm. }{ }^{11}\right) \\
S_{\mathfrak{n}} & =\exp \left\{\sum_{\mathfrak{f}_{v}}\left(f_{\mathfrak{t} v}(\mathfrak{n}) \beta_{\mathfrak{t} v}^{+}-f_{\mathfrak{t} v}^{*} \beta_{\mathfrak{t} v}\right),\right.
\end{aligned}
$$

11 Vgl. T. D. Lee, F. E. Low u. D. Pines, Phys. Rev. 90, 297 [1953]. 


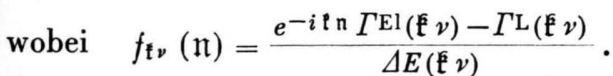

Mit den neuen Wellenfunktionen $\left.\left.\mid \mathfrak{n}^{\prime}\right)=S_{\mathfrak{n}} \mid \mathfrak{n}\right)$ erhalten wir dann für $\bar{H}$

$$
\begin{aligned}
& \bar{H}=\sum_{\mathrm{n} \mathfrak{n}} U^{*}(\mathfrak{n}) U(\overline{\mathfrak{n}})<_{\mathfrak{n}}^{\Re}\left|H_{E}\right|_{\frac{\mathfrak{n}}{R}}>\left(\mathrm{n}^{\prime}\left|S_{\mathfrak{n}} S_{\mathfrak{n}}^{-1}\right| \overline{\mathfrak{n}}^{\prime}\right) \\
& +\sum_{\mathrm{n}}|U(\mathfrak{n})|^{2}\left(\mathfrak{n}^{\prime}\left|\sum_{\mathfrak{\mathrm { f }} v} \Delta E(\mathfrak{f} v) \beta_{\mathfrak{\ddagger} v}^{+} \beta_{\mathfrak{\ddagger} v}\right| \mathfrak{n}^{\prime}\right) \\
& +\sum_{n}|U(\mathfrak{n})|^{2} \mathcal{V}(\mathfrak{n})
\end{aligned}
$$

Dabei ist

$$
\begin{aligned}
\mathcal{V}(\mathfrak{n}) & =-\sum_{\mathfrak{\ell} v}\left|f_{\mathfrak{k} v}(\mathfrak{n})\right|^{2} \Delta E(\mathfrak{f} v) \\
& =-\sum_{\mathfrak{k} \nu} \frac{\left|e^{-i \mathfrak{k} n} \Gamma^{\mathrm{El}}(\mathfrak{k} v)-\Gamma^{\mathrm{L}}(\mathfrak{k} v)\right|^{2}}{\Delta E(\mathfrak{k} v)} .
\end{aligned}
$$

Von der Wahl der Funktionen $\left.\mid \mathfrak{n}^{\prime}\right)$ hängen der zweite Term und die nichtdiagonalen Anteile $(\mathfrak{n} \neq \overline{\mathfrak{n}})$ des ersten Termes von (19) ab. Der zweite Term nimmt sein Minimum an für $\left.\mid \mathfrak{n}^{\prime}\right)=\Phi_{0}$. Mit dieser Wahl der $\left.\mid \mathfrak{n}^{\prime}\right)$, bei der wir die Matrixelemente $\left(\mathfrak{n}^{\prime}\left|S_{\mathfrak{n}} S_{\mathfrak{n}}^{-1}\right| \overline{\mathfrak{n}}^{\prime}\right)$ in guter Näherung gleich 1 setzen können, kommen wir auch dem Minimum des gesamten Funktionals $\bar{H}$ sehr nahe (s. Anhang 2). $\mathcal{V}(\mathfrak{n})$ ist dann das oben erwähnte $\mathrm{WW}$-Potential zwischen Elektron und Loch.

Wir berechnen $\mathcal{V}(\mathfrak{n})$. Mit (9) ergibt sich

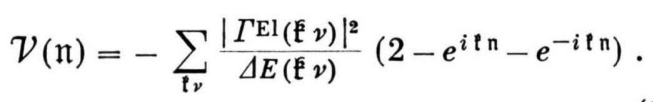

Für die $\Gamma^{\mathrm{El}}$ benutzen wir die Näherungsformel (12). Die Summe über $\mathfrak{f}$ wird in ein Integral über die erste BRILlouinsche Zone des kubisch flächenzentrierten Gitters verwandelt, die wir durch eine Kugel vom Radius $k_{\max }$ ersetzen; $4 \pi k_{\max }^{3} / 3=(2 \pi)^{3} \sigma$. Für $\Delta E(\mathfrak{f} \|)$ setzen wir $\Delta E(0 \|) \equiv \Delta E$ in die Summe ein und vernachlässigen die ${ }^{4}$-Abhängigkeit ${ }^{12}$. Die Integration ergibt

$\mathcal{V}(n)=-16 e^{2} \sigma \frac{p^{2}}{\Delta E}\left[k_{\max }-\frac{1}{n} \mathrm{Si}\left(n k_{\max }\right)\right](n \neq 0)$, $\mathcal{V}(0)=0$;

$\mathrm{Si}(x)$ ist der Integralsinus. Für $n \neq 0$ erhalten wir einen vom Abstand von Elektron und Loch unabhängigen Term und einen im wesentlichen mit $1 / n$ variierenden Term ${ }^{13}$. Dieser abstandsabhängige

12 Siehe Anhang 1.
Term liefert für die optische DK

$$
1-\left(1 / \varepsilon_{\text {opt }}\right)=8 \pi \sigma\left(p^{2} / \Delta E\right) .
$$

Das Ergebnis stimmt mit dem von Toyozawa ${ }^{1}$ sowie Haken und Sсноттку 2 überein, die die WW mit den Paarzuständen für große Ladungsabstände behandelt haben. Für den abstandsunabhängigen Term $\mathcal{V}_{\text {const }}$ ergibt sich

$\mathcal{V}_{\text {const }}=-\left(1-\frac{1}{\varepsilon_{\text {opt }}}\right) \frac{2}{\pi} e^{2} k_{\max }$

$$
=-5,5 \mathrm{eV} \text { für } \mathrm{NaCl} \text {. }
$$

(Dabei wurde der experimentelle Wert $\varepsilon_{\text {opt }}=2,25$ für $\mathrm{NaCl}$ eingesetzt.)

Beim vom Abstand abhängigen Anteil von $\mathcal{V}(n)$ spielen für große $n$ wegen der für große $k$ stark schwankenden Exponentialfunktion in (21) nur kleine $k$ eine Rolle. Die Verwendung der nur für kleine $k$ richtigen $\Gamma^{\mathrm{El}}$ ist dann gerechtfertigt. Für kleinere Ladungsabstände und vor allem für den abstandsunabhängigen Term sind auch größere $k$ wichtig.

\section{Der Einfluß von Paarzuständen mit großen Impulsen}

Bei der Berechnung des WW-Potentials $\mathcal{V}(n)$ sollen die Paarzustände mit großen Impulsen genauer berücksichtigt werden. Zunächst betrachten wir die abstandsunabhängige Polarisationsenergie

$$
\mathcal{V}_{\text {const }}=-2 \sum_{\mathfrak{P}_{\nu}} \frac{\mid \Gamma^{\left.\mathrm{El}(\mathfrak{k} v)\right|^{2}}}{\Delta E(\mathfrak{E} v)} .
$$

A. Wir benutzen für $\Gamma^{\mathrm{El}}$ die Integralapproximation (11). Die Summe über $\mathfrak{f}$ wird wie oben in ein Integral über die durch eine Kugel angenäherte erste Brillouinsche Zone verwandelt und $\Delta E(\mathfrak{f} \|)$ wird durch $\Delta E$ ersetzt. Die Integration ergibt [wir benutzen noch Gl. (23) ]

$$
\begin{aligned}
\mathcal{V}_{\text {const }}= & -\left(1-\frac{1}{\varepsilon_{\text {opt }}}\right) \frac{1}{\pi} \frac{e^{2}}{R_{0}} \\
& \cdot\left[\frac{\cos 2 R_{0} k_{\max }}{R_{0} k_{\max }}+2 \mathrm{Si}\left(2 R_{0} k_{\max }\right)-\frac{1}{R_{0} k_{\max }}\right] \\
= & -3,1 \mathrm{eV} \text { für } \mathrm{NaCl} .
\end{aligned}
$$

Der geringere Einfluß der größeren $k$ macht sich in diesem Ergebnis gegenüber dem im vorigen $\mathrm{Ab}$ schnitt erhaltenen von $-5,5 \mathrm{eV}$ (für $\mathrm{NaCl}$ ) deutlich bemerkbar.

B. Wir gehen auf die Darstellung (9) der $\Gamma^{\mathrm{El}}$

13 Bis auf die Schwankungen des Integralsinus ( $n k_{\max } \geq$ $n_{0} k_{\max }=4,3$ für $\left.\mathrm{NaCl}\right)$. 
durch Gittersummen zurück. Die Verwandlung einer Summe über die Gitterpunkte $m$ in ein Integral könnte vor allem in bezug auf die Exponentialfunktion bedenklich erscheinen. Wir benutzen deshalb die von Born und Bradburn ${ }^{14}$ mit Hilfe von Thetafunktionen durchgeführte Berechnung von Gittersummen.

$$
\Gamma^{\mathrm{El}}(\mathfrak{f} v)=\frac{e}{\sqrt{N}} \mathfrak{p}_{v} \cong ; \quad \subseteq=\sum_{\mathfrak{m} \neq 0} e^{i \mathfrak{f m}} \frac{\mathrm{m}}{|\mathfrak{m}|^{3}} .
$$

Wir führen dimensionslose Variable ein: $\mathfrak{\mathfrak { l }} a=\hat{\mathfrak{f}}$, $\mathfrak{m}=\hat{\mathfrak{m}} a, 2 a$ ist die Gitterkonstante.

$$
\widetilde{S}=-\frac{i}{a^{2}} \operatorname{grad} \hat{\mathfrak{\mathrm { f }}} \sum_{\hat{\mathfrak{m}} \neq 0} e^{i \hat{\mathrm{\ell}} \hat{\mathrm{m}} / \hat{m}^{3}} .
$$

Die hinter dem Gradienten stehende Gittersumme wurde von Born und BRADBURN für das kubisch flächenzentrierte Gitter berechnet. Es ergibt sich für $\subseteq$ (höhere Glieder sind vernachlässigt worden)

$$
\begin{aligned}
\widetilde{S}=-\frac{i \pi}{a^{2}}[ & 0,17 \operatorname{grad}_{\hat{\mathrm{t}}}\left(\cos \hat{k_{1}} \cos \hat{k_{2}}+\cos \hat{k_{1}} \cos \hat{k_{3}}\right. \\
& \left.\left.+\cos \hat{k_{2}} \cos \hat{k_{3}}\right)-2 \frac{\hat{\mathfrak{k}}}{\hat{k}^{2}} e^{-\hat{k}^{2} / \pi}\right] .
\end{aligned}
$$

Während der zweite Term in der eckigen Klammer immer longitudinal ist $(\| \hat{\mathfrak{f}})$, ist der erste Term nicht mehr immer longitudinal. Es tragen also in Strenge auch transversale Paarzustände zur WW bei. Der longitudinale zweite Term allein liefert ca. $70 \%$ von $\mathcal{V}_{\text {const }}$. Den ersten Term werten wir in Richtungen aus, in denen er longitudinal ist.
a) $\hat{k}_{2}=\hat{k}_{3}=0$
usw. Würfelkante,
b) $\hat{k}_{1}=\hat{k}_{2}, \quad \hat{k}_{3}=0$ usw. Flächendiagonale,
c) $\hat{k}_{1}=\hat{k}_{2}=\hat{k}_{3} \quad$ usw. Raumdiagonale.

Die Werte für eine solche ausgezeichnete Richtung übertragen wir jeweils auf den gesamten $\hat{f}$-Raum. $\mathcal{V}_{\text {const }}$ wird dann wieder wie oben berechnet, indem die Summe über $\mathfrak{f}$ durch ein Integral ersetzt wird. Numerische Integration ergibt
a) $\mathcal{V}_{\text {const }}=-1,04$ )
$\left.\begin{array}{ll}\text { a) } & \mathcal{V}_{\text {const }}=-1,04 \\ \text { c) } & \mathcal{V}_{\text {const }}=-1,02 \\ \text { const } & =-0,95\end{array}\right\} \cdot\left(1-\frac{1}{\varepsilon_{\text {opt }}}\right) \frac{e^{2}}{a}$.

Für NaCl erhält man dann (über alle drei Fälle gemittelt) $\mathcal{V}_{\text {const }}=-2,8 \mathrm{eV}$.

$\mathrm{Zu}$ dieser elektronischen Polarisationsenergie

14 M. Born u. M. Bradburn, Proc. Cambr. Phil. Soc. 39, 104 [1943] und frühere Arbeiten. kommt noch der Anteil hinzu, der von den optischen Ionenschwingungen herrührt. Dieser Anteil ist $\mathcal{W}_{\text {const }}=2 \hbar \omega g \approx 0,3 \mathrm{eV}$ für $\mathrm{NaCl}$ ( $g$ ist die Kopplungskonstante für optische Ionenschwingungen ${ }^{15}$ ).

C. Bei Verwendung der Pol-Dipol-Näherung für $\Gamma^{\mathrm{El}}$ und $\Gamma^{\mathrm{L}}[\mathrm{Gl}$. (9)] ergibt sich keine WW mit den Paarzuständen, wenn sich Elektron und Loch am gleichen Halogenion befinden. Wir schätzen

$$
\mathcal{V}(0)=-\sum_{\mathfrak{i} v} \frac{\left|\Gamma^{\mathrm{El}}(\mathfrak{k} v)-\Gamma^{\mathrm{L}}(\mathfrak{k} v)\right|^{2}}{\Delta E(\mathfrak{k} v)}
$$

mit Gl. (10) für $\Gamma^{\mathrm{El}}-\Gamma^{\mathrm{L}}$ ab. Dazu benötigen wir die Ausdehnungen der WanNiER-Funktionen. Leider steht

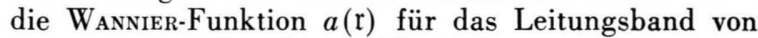
$\mathrm{NaCl}$ (und der anderen Alkalihalogenide) nicht zur Verfügung. Tiвbs ${ }^{16}$ hat die Bцoch-Funktion für $\mathfrak{k}=0$ des Leitungsbandes von $\mathrm{NaCl}$ in 100-Richtung berechnet. Zur Abschätzung von $\mathcal{V}(0)$ machen wir die folgende allerdings grobe Näherung. Die BцocH-Funktionen werden in der Form $e^{i \mathfrak{f} r} u_{\mathfrak{p}}(\mathrm{r})$ geschrieben und die Ł-Abhängigkeit des gitterperiodischen Teils wird vernachlässigt, d. h. $u_{\mathfrak{p}}(\mathrm{r})=u_{0}(\mathrm{r})$ gesetzt. Dann kann die WanNiER-Funktion in 100-Richtung berechnet und in die übrigen Richtungen extrapoliert werden. Für die Ausdehnung dieser Funktion ergibt sich

$$
\overline{r^{2}}=\int r^{2}|a(\mathrm{r})|^{2} \mathrm{~d} \tau \approx 13{a_{0}}^{2} \text { für } \mathrm{NaCl} .
$$

Auf den wesentlich geringeren Beitrag des Loches wurde im Rahmen dieser Abschätzung verzichtet. Die Gittersumme über $m$ in Gl. (10) wurde nach BoRN und Bradburn ermittelt und $\mathcal{V}(0)$ dann analog der Rechnung in $\mathbf{B}$. berechnet. Es ergibt sich

$$
\mathcal{V}(0)=-0,38\left(1-\frac{1}{\varepsilon_{0 \mathrm{pt}}}\right) \frac{e^{2}}{a}\left(\overline{r^{2}}\right)^{2} \approx-0,2 \mathrm{eV}
$$

für $\mathrm{NaCl}$.

$\mathcal{V}(0)$ ist vernachlässigbar klein gegen die im betrachteten Fall auftretende Couloмв-Energie.

D. Der geringere Einfluß von Paarzuständen mit großen Impulsen bei Verwendung von Gl. (11) gegenüber Gl. (12) für $\Gamma^{\mathrm{El}}$ macht sich auch beim abstandsabhängigen Anteil von $\mathcal{V}(n)$ bemerkbar. Die geringere Wirkung größerer $k$ kompensiert die in Gl. (22) vorhandenen Schwankungen des Integralsinus. Wir schreiben das sich mit Gl. (11) ergebende $\mathcal{V}(n)$ in der zu Gl. (22) analogen Form

$$
\begin{array}{r}
\mathcal{V}(n)=\left(1-\frac{1}{\varepsilon_{\mathrm{opt}}}\right) \frac{2}{\pi} \frac{1}{n} S\left(n k_{\max }\right)+\mathcal{V}_{\text {const }} \\
(n \neq 0) .
\end{array}
$$

Die hier eingeführte Funktion $S\left(n k_{\max }\right)$ schwankt für die ersten vier Abstände nur noch zwischen 1,54 und 1,59 (Grenzwert $\pi / 2$ ), während der Integralsinus im gleichen Bereich zwischen 1,43 und 1,69 schwankt.

\footnotetext{
15 T. D. Lee, F. E. Low u. D. Pines, Phys. Rev. 90, 197 [1953]. - H. Haken, Z. Phys. 146, 527 [1956].

16 S. R. Tibis. Trans. Faraday Soc. 35, 1471 [1939].
} 


\section{Die Lorentz-Korrektur für die optische DK}

Wir betrachten Gl. (23) für die optische DK, schreiben den in $\Delta E$ enthaltenen Dipolterm explizit an ${ }^{17}$ und führen die Polarisierbarkeit pro Halogenion im Kristall $\alpha=2 p^{2} / \overline{\Delta E}$ ein $^{18}$.

$$
\left(1-\frac{1}{\varepsilon_{\mathrm{opt}}}\right)=\frac{4 \pi \sigma \alpha}{1+4 \pi \sigma \alpha / 3} .
$$

Da für große Ladungsabstände nur Paarzustände mit sehr kleinen $k$ zum abstandsabhängigen Anteil der Polarisationsenergie beitragen, verschwinden die durch Vernachlässigung des Rückstoßes und Ersetzung von $\Delta E(\mathfrak{f} \|)$ durch $\Delta E$ entstehenden Fehler für Gl. (34).

Dexter und Heller ${ }^{19}$ behandeln die WW von Lichtwellen mit transversalen Paarzuständen. Ihre Rechnung liefert (bei Vernachlässigung von quadratischen Termen in $4 \pi \sigma \alpha / 3$ ) für die optische DK die Lonentz-Korrektur

$$
\varepsilon_{\mathrm{opt}}-1=\begin{gathered}
4 \pi \sigma a \\
1-4 \pi \sigma a / 3
\end{gathered} .
$$

Die aus den Gln. (34) und (35) für $\varepsilon_{\text {opt }}$ folgenden Werte stimmen nicht überein. Wir wollen zeigen, daß sich mit einem verbesserten WW-Glied auch in dem hier betrachteten Modell (WW von Ladungen mit longitudinalen Paarzuständen) die LoRentzKorrektur für $\varepsilon_{\text {opt }}$ ergibt. Wie Dexter und Heller vernachlässigen wir quadratische Terme in $4 \pi \sigma \alpha / 3$.

Wir haben beim Hamilton-Operator [Gln. (6) und (7)] die Emission (bzw. Absorption) eines Paarzustandes berücksichtigt. Der Coulomb-Operator läßt aber auch die simultane Emission (bzw. Absorption) zweier Paarzustände zu. Wir erweitern den Hamilton-Operator $H$ um die Terme

$$
\frac{1}{2} \sum_{\mathbf{i}} \gamma_{\mathfrak{f}} \beta_{\mathbf{t} \|}^{+} \beta_{-\mathbf{f} \|}^{+}+\text {(herm. konj.). }
$$

Wir haben nur longitudinale Paarzustände berücksichtigt. Die Terme (36) beschreiben die Emission (bzw. Absorption) zweier Paarzustände mit entgegengesetzten Impulsen. (Der Impuls des Exzitons wird von diesen Prozessen nicht berührt.) Die Faktoren $\frac{1}{2}$ stehen vor den Summen, weil bei der Summation über $\mathfrak{f}$ jeder Prozeß doppelt auftritt. Die $\gamma_{\mathrm{f}}$ sind die entsprechenden Matrixelemente des Cousomb-

17 Siehe Anhang 1.

18 Diese Beziehung für die Polarisierbarkeit $a$ wird bei Erweiterung des Modells abgeändert. Bei Berücksichtigung des Spins (Exziton und Paarzustände im Singulettzustand) kommt ein Faktor 2 hinzu, bei Berücksichtigung mehrerer

\section{Operators}

$$
\begin{aligned}
\gamma_{\mathfrak{t}}=\frac{1}{N} \sum_{\mathrm{m}} e^{-i \mathfrak{l} \mathrm{m}} \int a^{*}\left(\mathrm{r}_{1}\right) a^{*}\left(\mathrm{r}_{2}-\mathfrak{m}\right) \frac{e^{2}}{\left|\mathrm{r}_{1}-\mathrm{r}_{2}\right|} \\
\cdot a_{||}\left(\mathrm{r}_{1}\right) a_{||}\left(\mathrm{r}_{2}-\mathfrak{m}\right) \mathrm{d} \tau_{12} .
\end{aligned}
$$

Die $\gamma_{\mathrm{t}}$ sind formal identisch mit den Dipoltermen von Heller und Marcus ${ }^{20}$.

Die Berechnung des WW-Potentials erfolgt in gleicher Weise wie im Abschnitt 2. Die Terme (36) treten zu $H_{\mathrm{P}}$ (n) [Gl. (16) ] hinzu.

$$
\begin{aligned}
H_{\mathrm{P}}(\mathfrak{n})=\sum_{\mathfrak{l}} & C_{\mathfrak{f}}(\mathfrak{n}) \beta_{\mathfrak{t} \|}^{+}+\frac{1}{2} \sum_{\mathfrak{p}} \gamma_{\mathfrak{t}} \beta_{\mathfrak{f} \|}^{+} \beta_{-\mathfrak{f} \|}^{+} \\
& +(\text {herm. konj. })+\sum_{\mathfrak{t}} \Delta E(\mathfrak{f} \|) \beta_{\mathfrak{f} \|}^{+} \beta_{\mathfrak{f} \|} .
\end{aligned}
$$

$C_{\mathfrak{f}}(\mathfrak{n})=e^{-i \mathfrak{l n}} \Gamma^{\mathrm{El}}(\mathfrak{f} \|)-\Gamma^{\mathrm{L}}(\mathfrak{f} \|)$.

Wir gehen mit Hilfe einer kanonischen Transformation $S$ zu neuen Operatoren $\delta_{\mathrm{e}}$ über.

$$
\begin{aligned}
& S=1-\frac{1}{2} \sum_{\mathfrak{f}} \bar{\gamma}_{\mathfrak{t}} \beta_{\mathfrak{f} \|}^{+} \beta_{-\mathfrak{t} \|}^{+}+\frac{1}{2} \sum_{\mathfrak{f}} \bar{\gamma}_{\mathfrak{f}} \beta_{\mathfrak{t} \|} \beta_{-\mathfrak{t} \|}, \\
& \delta_{\mathfrak{t}}=S \beta_{\mathfrak{t} \|} S^{-1}=\beta_{\mathfrak{t} \|}+\beta_{-\mathfrak{t} \|}^{+} \bar{\gamma}_{\mathfrak{t}}, \quad \gamma_{\mathfrak{t}}=\gamma_{\mathfrak{t}} / 2 \Delta E(\mathfrak{f} \|) .
\end{aligned}
$$

$S$ ist unitär bei Vernachlässigung von quadratischen Termen in $\bar{\gamma}_{\hat{t}}$. Wir betrachten die $\bar{\gamma}_{\mathrm{f}}$ als reell, da wir die Dipolmomente $\mathfrak{p}_{\|}$als reell ansehen ${ }^{21}$. Wir schreiben $H_{\mathrm{P}}(\mathfrak{n})$ auf die neuen Operatoren um und erhalten bei Vernachlässigung von quadratischen Termen in $\bar{\gamma}_{\mathrm{f}}$

$$
\begin{aligned}
H_{\mathrm{P}}(\mathfrak{n})= & \sum_{\mathfrak{f}} C_{\mathfrak{f}}\left(1-\gamma_{\mathfrak{f}}\right) \delta_{\mathfrak{f}}^{+}+\text {(herm. konj.) } \\
& +\sum_{\mathfrak{f}} \Delta E(\mathfrak{f} \|) \delta_{\mathfrak{t}}^{+} \delta_{\mathfrak{f}} .
\end{aligned}
$$

Die Berücksichtigung der simultanen Emission und Absorption zweier Paarzustände führt in unserer Näherung dazu, daß die Glieder für Emission (bzw. Absorption) eines Paarzustandes mit dem Faktor $\left(1-\bar{\gamma}_{\mathrm{t}}\right)$ multipliziert werden. Da diese WW-Glieder (bei Integralapproximation) nur für longitudinale Paarzustände vorhanden sind, liefert die Berücksichtigung transversaler Paarzustände auch hier keinen Beitrag. Der Einfluß auf die optische DK ist nun sofort zu übersehen. Jeder Term in $\mathcal{V}(n)$ [Gl.(20)] wird mit $\left(1-\bar{\gamma}_{n}\right)^{2}$ multipliziert. Da für den abstandsabhängigen Anteil der Polarisationsenergie nur Paarzustände mit kleinen $k$ beitragen, können

angeregter Zustände summieren sich die Polarisierbarkeiten.

19 D. L. Dexter u. W. R. Heller, Phys. Rev. 91, 273 [1953].

20 W. R. Heller u. A. Marcus, Phys. Rev. 84, 809 [1951].

21 Siehe Anhang 1. 
wir in $\bar{\gamma}_{\mathfrak{i}}$ den Impuls $\mathfrak{f}=0$ setzen. Nach Heller und Marcus ist für longitudinale Paarzustände $\bar{\gamma}_{0}=2 \pi \sigma \alpha / 3$ (s. Anm. ${ }^{20,}{ }^{21}$ ). An Stelle von Gl. (34) ergibt sich damit für die optische DK

$$
1-\frac{1}{\varepsilon_{\mathrm{opt}}}=\frac{4 \pi \sigma \alpha}{1+4 \pi \sigma \alpha / 3}\left(1-\frac{2 \pi \sigma \alpha}{3}\right)^{2} .
$$

Gl. (41) liefert (bei Vernachlässigung quadratischer Terme in $4 \pi \sigma \alpha / 3)$ die Lonentz-Korrektur. In Abb. 1 sind die für $\varepsilon_{\text {opt }}$ aus den Gln. (34), (35) und (41) folgenden Werte in Abhängigkeit von

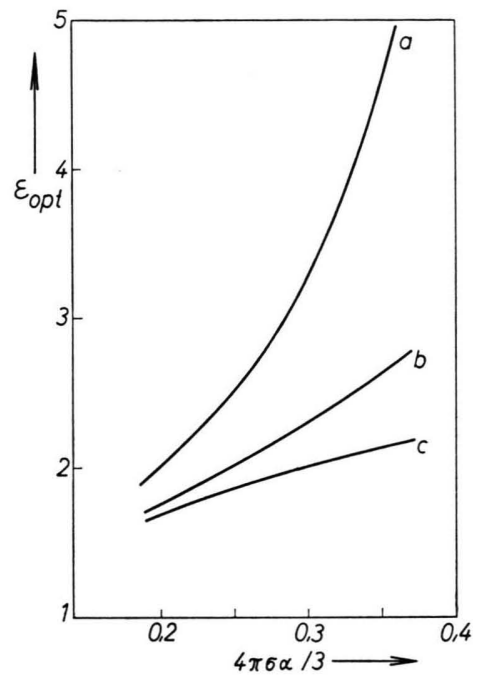

Abb. 1. $\varepsilon_{\text {opt }}$ in Abhängigkeit von $4 \pi \sigma \alpha / 3$. a) $\varepsilon_{0 p t}$ unkorrigiert nach Gl. (34); b) $\varepsilon_{\text {opt }}$ mit LoREnTz-Korrektur nach Gl. (35) ; c) $\varepsilon_{\text {opt }}$ korrigiert nach Gl. (41).

$4 \pi \sigma \alpha / 3$ aufgetragen. Für die meisten Alkalihalogenide liegt nach $\mathrm{Gl}$. (35) $4 \pi \sigma \alpha / 3$ zwischen 0,2 und 0,3 (für $\mathrm{NaCl} 0,3$ ). Eine Berücksichtigung quadratischer und höherer Terme in $4 \pi \sigma \alpha / 3$ wäre wünschenswert, dürfte jedoch ebenso wie im Fall transversaler Paarzustände große Schwierigkeiten bieten.

\section{Diskussion}

In Abb. 2. ist das von der elektronischen Polarisation erzeugte Potential $\mathcal{V}(n)$ zwischen Elektron

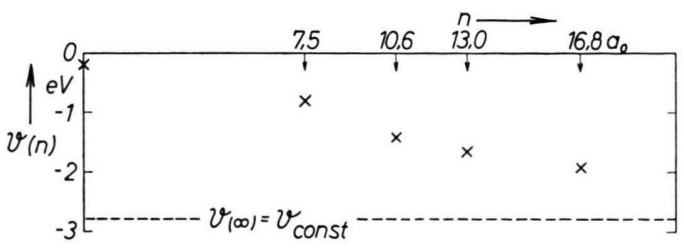

Abb. 2. $\mathcal{V}(n)$ für $\mathrm{NaCl}$. und Loch nach den Ergebnissen der Abschnitte 2 und 3 für $\mathrm{NaCl}$ aufgetragen.

In Anwendung auf Exzitonmodelle ergeben sich die nachstehenden Folgerungen.

A. Für $n>0$ haben wir $\mathcal{V}(n)$ aufgeteilt in einen abstandsunabhängigen Anteil $\mathcal{V}_{\text {const }}=\mathcal{V}(\infty)$ und einen abstandsabhängigen Anteil. Der abstandsabhängige Anteil der Polarisationsenergie rechtfertigt die Einfügung der optischen DK ins Couloms-Gesetz.

B. Für den Exzitonanteil, bei dem sich Elektron und Loch am gleichen Ion befinden, ist die Polarisationsenergie vernachlässigbar. Eine Aufteilung in $\mathcal{V}_{\text {const }}$ und einen übrigen Anteil erscheint dabei physikalisch nicht begründet. Für die Coulomb-Energie und die hier noch auftretende Austauschenergie ist $\varepsilon=1 \mathrm{zu}$ verwenden. Der bei dem Modell von Muro und Окимо ${ }^{5}$ für die Соuцомв-Energie benutzte volle Wert der optischen DK ist nicht berechtigt. Dagegen wird die Coulomв-Energie durch die Austauschenergie zwischen Elektron und Loch verringert.

C. Die Polarisationsenergie für ein Loch im Valenzband und ein davon weit entferntes Elektron im Leitungsband ist $\mathcal{V}_{\text {const }}(-2,8 \mathrm{eV}$ für $\mathrm{NaCl})$ plus der von der Ionenpolarisation erzeugten Energie $\mathcal{W}_{\text {const }}(-0,3 \mathrm{eV}$ für $\mathrm{NaCl})$. Diese Polarisationsenergie vermindert den sich ohne Berücksichtigung der Polarisation ergebenden Bandabstand auf den experimentellen Wert (9,5 eV für $\mathrm{NaCl})$.

Bei Exzitonen in Alkalihalogeniden dominiert (für den Grundzustand) der Exzitonanteil, bei dem sich Elektron und Loch am gleichen Ion befinden. Bei der Berechnung der Energie des Exzitons kommt dann für diesen Exzitonanteil der Energiebetrag $\left|\mathcal{V}_{\text {const }}+\mathcal{W}_{\text {const }}\right|$ hinzu, wenn man durchweg den experimentellen Bandabstand benutzt.

Herrn Prof. Dr. Kockel möchte ich für sein Interesse an dieser Arbeit danken.

\section{Anhang 1}

Für die Matrixelemente von $H_{\mathrm{E}}$ ergibt sich in unserer Darstellung 7, 22

$$
\begin{aligned}
\left\langle\begin{array}{c}
\Omega \\
\mathfrak{n}
\end{array} H_{E} \mid \frac{\Omega}{\mathfrak{n}}\right\rangle & \text { (A l) } \\
= & \varepsilon_{\mathrm{L}}(\mathfrak{n}-\mathfrak{n})-e^{i \Re(\mathfrak{n}-\mathfrak{n})} \varepsilon_{\mathrm{V}}(\mathfrak{n}-\overline{\mathfrak{n}})-C(\mathfrak{n}) \delta_{\mathfrak{n} \mathfrak{n}} \\
& +2\left[A(0)+\sum_{\mathfrak{n} \neq 0} A(\mathfrak{m}) e^{i \Re \mathfrak{m}}\right] \delta_{\mathfrak{n}, 0} \delta_{\overline{\mathfrak{n}}, 0 .}
\end{aligned}
$$

22 Y. TAkeuti, Progr. Theor. Phys. 18, 421 [1957]. 
Dabei sind

$$
\begin{aligned}
& C(\mathfrak{n})=\int a^{*}\left(\mathrm{r}_{1}-\mathfrak{n}\right) a\left(\mathrm{r}_{1}-\mathfrak{n}\right) \frac{e^{2}}{\left|\mathrm{r}_{1}-\mathrm{r}_{2}\right|} \\
& \cdot a_{\mu}^{*}\left(\mathrm{r}_{2}\right) a_{\mu}\left(\mathrm{r}_{2}\right) \mathrm{d} \tau_{12}, \\
& A(\mathfrak{n})=\int a^{*}\left(\mathrm{r}_{1}-\mathfrak{n}\right) a_{\mu}\left(\mathrm{r}_{1}-\mathfrak{n}\right) \frac{e^{2}}{\left|\mathrm{r}_{1}-\mathrm{r}_{2}\right|} \\
& \cdot a_{\mu}^{*}\left(\mathrm{r}_{2}\right) a\left(\mathrm{r}_{2}\right) \mathrm{d} \tau_{12}
\end{aligned}
$$

die Coulomb- und Austauschintegrale.

In Gl. (A 1) wurde der Spin der Elektronen berücksichtigt. Die Formel ist für den Singulettzustand des Exzitons aufgeschrieben, die Austauschterme (in der eckigen Klammer) treten mit einem Faktor 2 auf. Der zweite Term in der eckigen Klammer ist der Dipolterm von Heller und Marcus ${ }^{20}$. $\varepsilon_{\mathrm{V}}(\mathrm{n})$ und $\varepsilon_{\mathrm{L}}(\mathrm{n})$ sind die Fourier-Komponenten der Bloch-Energien für das Valenzband und das Leitungsband ${ }^{7,23}$. Die $\varepsilon_{\mathrm{V}}(\mathfrak{n})$ hängen für $\mathfrak{n} \neq 0$ von der Orientierung $\mu$ des Exzitons ab. Bei einem wenig ausgedehnten Exziton wird sich die Orientierung nicht nach den Valenzbandenergien $\varepsilon_{\mathrm{V}}$ (im Gegensatz zur Orientierung eines Valenzelektrons), sondern nach dem Dipolterm richten; d. h. die Orientierung wird longitudinal oder transversal zum Impuls $R$ sein.

Die Energie eines Paarzustandes ist

$$
\Delta E(\mathfrak{k} v)=\left\langle\begin{array}{l}
\mathfrak{k} \\
0
\end{array}\left|H_{E}\right| \begin{array}{l}
\mathfrak{t} \\
0
\end{array}\right\rangle=\overline{\Delta E}+2 \sum_{\mathfrak{m} \neq 0} A(\mathfrak{m}) e^{i \mathfrak{l} \mathrm{m}},
$$

wobei die Orientierung $\mu$ des Exzitons durch $\nu$ zu ersetzen ist. Die Behandlung des Dipolterms nach Heller und Marcus ${ }^{20}$ ergibt

$$
\begin{aligned}
& \sum_{\mathfrak{m} \neq 0} A(\mathfrak{m}) e^{i \mathfrak{l} \mathrm{m}} \\
& \quad=\frac{8 \pi \sigma}{3} \mathfrak{p}_{v}^{2} P_{2}\left[\cos \left(\mathfrak{p}_{v}, \mathfrak{k}\right)\right]\left\{j_{0}\left(k R_{0}\right)+j_{2}\left(k R_{0}\right)\right\} .
\end{aligned}
$$

Dabei wurden die Dipolmomente $\mathfrak{p}_{v}$ als reell behandelt. $P_{2}$ ist das Legendresche Polynom 2. Ordnung, $j_{0}$ und $j_{2}$ sind sphärische BESSEL-Funktionen. Damit erhalten wir

$$
\Delta E \equiv \Delta E(0 \|)=\overline{\Delta E}(1+4 \pi \sigma \alpha / 3) ;
$$

$a$ ist wieder die Polarisierbarkeit pro Halogenion. Der bei Berücksichtigung des Spins bei dem Dipolterm auftretende Faktor 2 geht in die Polarisierbarkeit ein ${ }^{18}$.

Wir haben in Abschnitt 2 die $\mathfrak{k}$-Abhängigkeit von $\Delta E(\mathfrak{E} \|)$, d. h. die $\mathfrak{k}$-Abhängigkeit des Dipolterms vernachlässigt. $\Delta E(0 \|)$ hat etwa die Größe der Energielücke zwischen Valenz- und Leitungsband plus abstandsunabhängiger Polarisationsenergie $(\approx 13 \mathrm{eV}$ für NaCl$)$; die Vernachlässigung beim Dipolterm beträgt maximal $\approx 2 \mathrm{eV}$ für $\mathrm{NaCl}$, ist jedoch (auch wegen der Bevorzugung von Paarzuständen mit kleinen Impulsen) im Mittel geringer.

23 G. F. Koster u. J. C. Slater, Phys. Rev. 95, 1167 [1954].

24 Bei Berücksichtigung der §-Abhängigkeit der Phasen der
Für die Vernachlässigung des Rückstoßes auf das Exziton bei Absorption oder Emission von Paarzuständen kommen nur die $\Re$-abhängigen Anteile von $\left\langle\begin{array}{c}\mathcal{R} \\ \mathfrak{n}\end{array}\left|H_{E}\right| \begin{array}{l}\mathcal{R} \\ \mathfrak{n}\end{array}\right\rangle$ in Betracht. Wegen $\left.S_{0}=1 ; \mid 0\right)=\Phi_{0}$ tritt beim Dipolterm kein Rückstoß auf. Nach Gl. (A 1) ist dann die Vernachlässigung des Rückstoßes maximal gleich der Breite des Valenzbandes $(\approx 1,5 \mathrm{eV}$ für $\mathrm{NaCl})$; im Mittel ist sie (auch wegen der Bevorzugung von Paarzuständen mit kleinen Impulsen) geringer ${ }^{24}$.

\section{Anhang 2}

Wir diskutieren die Wahl der Funktionen $\left.\mid \mathfrak{n}^{\prime}\right)=\Phi_{0}$. Die von der Wahl dieser Funktionen abhängigen Terme von Gl. (19) lauten

$$
\begin{array}{r}
W=\sum_{\mathfrak{n} \mathfrak{n}} U^{*}(\mathfrak{n}) U(\overline{\mathfrak{n}})\left[\varepsilon_{\mathrm{L}}(\mathfrak{n}-\overline{\mathfrak{n}})-\varepsilon_{\mathrm{V}}(\mathfrak{n}-\mathfrak{n}) e^{i \Re(\mathfrak{n}-\overline{\mathfrak{n}})}\right] \\
\cdot\left(\mathfrak{n}^{\prime}\left|S_{\mathfrak{n}} S_{\overline{\mathfrak{n}}}^{-1}\right| \mathfrak{n}^{\prime}\right) .
\end{array}
$$

Dabei wurde Gl. (A 1) benutzt. Das Funktional $W$ liefert den Anteil zur kinetischen Energie des Exzitons, der dem $W_{\text {Ansierschen Modell }}{ }^{25}$ entspricht. Dieser Anteil ist bei einem wenig ausgedehnten Exziton geringer als bei einem weit ausgedehnten "W $W_{\text {ANNIER- }}$ schen" Exziton. Wir beschränken uns auf nächste Nachbarn und kleine $K$. $\quad\left[\varepsilon_{\mathrm{L}}(\mathfrak{n})=-\varepsilon_{\mathrm{L}}<0, \varepsilon_{\mathrm{V}}(\mathfrak{n})=\varepsilon_{\mathrm{V}}\right.$ $>0$, falls $n$ nächste Halogenionen verbindet, sonst $\varepsilon_{\mathrm{L}}(\mathrm{n})=\mathcal{E}_{\mathrm{V}}(\mathrm{n})=0$.].

Das Funktional $W$ soll zunächst ohne die von den Paarzuständen herrührenden Matrixelemente diskutiert werden.

$$
W^{\prime}=-\sum_{\mathrm{n} \mathrm{n}_{0}} U^{*}\left(\mathrm{n}+\mathrm{n}_{0}\right) U(\mathrm{n})\left[\varepsilon_{\mathrm{L}}+\varepsilon_{\mathrm{V}} e^{i \Omega_{\mathrm{n}_{0}}}\right],
$$

$\mathfrak{n}_{0}$ verbindet nächste Halogenionen.

Wir entwickeln $e^{i \Re n_{0}}$ für kleine $K$ und bestimmen das Minimum von $W^{\prime}$ bezüglich der Phasen $\operatorname{der} U(\mathfrak{n})$. Wir können diese Phasen so wählen, daß jeder Term von $W^{\prime}$ negativ reell wird. Es ergibt sich

$$
\begin{aligned}
W^{\prime}=-\sum_{\mathrm{n} \mathrm{n}_{0}} \mid & U\left(\mathrm{n}+\mathrm{n}_{0}\right) U(\mathrm{n}) \mid \\
\cdot & {\left[\varepsilon_{\mathrm{L}}+\varepsilon_{\mathrm{V}}-\frac{1}{2} \frac{\varepsilon_{\mathrm{L}} \varepsilon_{\mathrm{V}}}{\varepsilon_{\mathrm{L}}+\varepsilon_{\mathrm{V}}}\left(\Omega \mathrm{n}_{0}\right)^{2}+\ldots\right] . }
\end{aligned}
$$

Der zweite Term in der eckigen Klammer entspricht dem $\mathbb{W}_{\text {ANNIERschen }}$ Anteil an der kinetischen Energie, denn $\varepsilon_{\mathrm{L}} \varepsilon_{\mathrm{V}} /\left(\varepsilon_{\mathrm{L}}+\varepsilon_{\mathrm{V}}\right)$ ist proportional $1 /\left(m_{1}+m_{2}\right)$.

Beim Funktional $W$ kann man in gleicher Weise vorgehen. Die Phasen der $U$ werden auch hier so gewählt, $\mathrm{da} ß$ alle Terme negativ reell werden. Es kommt dann für das Minimum von $W$ darauf an, den Betrag der Matrixelemente $\left(\mathrm{n}+\mathrm{n}_{0}^{\prime}\left|S_{\mathfrak{n}+\mathfrak{n}_{0}} S_{\mathfrak{n}}^{-1}\right| \mathfrak{n}^{\prime}\right)$ möglichst groß zu machen. Der Maximalwert 1 (für unitäre Operato-

Verteilungsfunktion $U$ wird die Rückstoßenergie noch verringert, vgl. Anhang 2.

25 G. H. Wannier, Phys. Rev. 52, 191 [1937]. 
ren) wird durch die Funktionen $\left.\mid \mathfrak{n}^{\prime}\right)=\Phi_{0}$ schon in guter Näherung erreicht. Für die Matrixelemente ergibt sich durch Entwickeln der $S$

$$
\begin{aligned}
\left(\left|S_{\mathfrak{n}+\mathfrak{n}_{0}} S_{\mathfrak{n}}^{-1}\right|\right)= & 1+\frac{1}{2} \frac{\mathcal{V}(n)}{\Delta E}+\ldots \\
& \approx 1-0,03 \text { für } \mathrm{NaCl} .
\end{aligned}
$$

Der durch unsere spezielle Wahl der Funktionen $\mid \mathbf{n}^{\prime}$ ) entstehende Fehler kann somit maximal $0,03 W^{\prime}$ betragen. $W^{\prime}$ ist für ein weit ausgedehntes W $W_{\text {ANNIERsches }}$ Exziton etwa gleich der halben Summe der Bandbreiten von Valenz- und Leitungsband und für ein wenig ausgedehntes Exziton wesentlich geringer. $0,03 W^{\prime}$ kann gegen $\mathcal{V}(n)$ vernachlässigt werden.

\title{
Zur Theorie des F-Zentrums*
}

\section{Atomistische Formulierung des НаміLтом-Operators und statische Elektron-Gitter-Kopplung

\author{
Von Max Wagner
}

\begin{abstract}
Aus dem Institut für theoretische und angewandte Physik der Technischen Hochschule Stuttgart
\end{abstract}
(Z. Naturforschg. 16 a, 410—426 [1961] ; eingegangen am 11. November 1960)

\begin{abstract}
Die in den beiden vorangehenden Untersuchungen ${ }^{1}$ kontinuumstheoretisch beschriebene Elektron-Gitter-Statik wird hier auf streng atomistischer Basis formuliert und dabei die „effektive“ Masse des Störstellenelektrons ausgeschieden. Die Wirkung eines Gitterions wird als Überlagerung von Punktladung und Polarisationsdipol erfaßt. Die Ionenpolarisation folgt der Bewegung des Störstellenelektrons momentan; da dessen Feld eine Singularität besitzt, wird eine Modifikation des (sonst linearen) Polarisationsgesetzes erzwungen. Der Hamilton-Operator - insbesondere der auf statisches Gleichgewicht reduzierte - wird explizit angegeben. Mit einer einfachen, einparametrigen Wellenfunktion, die der Überlagerung von zentraler und gitterperiodischer Symmetrie im HamiLtonOperator adäquat ist, wird der Grundzustand des $F$-Zentrums bei $\mathrm{KBr} z u-1,5 \mathrm{eV}$ berechnet.
\end{abstract}

In zwei vorangehenden Arbeiten ${ }^{1}$ wurde der Versuch unternommen, eine Theorie des $F$-Zentrums aufzubauen, die die dynamische Kopplung zwischen Gitter und Störstellenelektron („dynamische Elektron-Gitter-Kopplung“) in möglichst guter Anpassung an die physikalische Realität beschreibt, hinausgehend über die formale Beschreibungsweise der bisherigen Untersuchungen ${ }^{2}$, deren entgegengesetzt extreme Ausgangspositionen zu überbrücken sind.

$\mathrm{Da}$ in den Teilen I und II das Interesse auf dem dynamischen Wechselspiel lag, wurde dort die statische Elektron-Gitter-Kopplung nicht atomistisch, sondern durch eine Kontinuumstheorie gefaßt. Überdies enthalten die beiden vorangehenden Untersuchungen die effektive Masse des lokalisierten Elektrons als nichtlegitime Größe, die aus dem Experiment bestimmt werden muß.

In dieser Arbeit soll nun auch die statische Elektron-Gitter-Kopplung - der physikalischen Realität gemäß - atomistisch beschrieben und die effektive Masse des Elektrons aus der Theorie ausgeschieden werden, so daß die atomistische Behandlung des $F$-Zentrums als vollständig angesehen werden kann.

\footnotetext{
* Dissertation, Techn. Hochschule, Stuttgart 1960 (3. Teil)

1 M. W 302 [1961]; künftig mit I und II zitiert.
}

Das quantenmechanische Gesamtsystem - als solches gilt uns ein Mikrowürfel des Kristalls mit einem einzigen $F$-Zentrum - kann anschaulich in drei Untersysteme zerlegt werden: 1. Das System der Gitterelektronen, welche die Ionenhüllen aufbauen. 2. Das Störstellenelektron. 3. Die Atomkerne des Gitters. Mathematisch kann diese Zerlegung, wie in Teil I ausführlich dargelegt, durch die beiden adiabatischen Näherungen realisiert werden. Bliebe man nun weiterhin quantenmechanisch streng legitim, so wären drei SchröDIngER-Gleichungen in festgelegter Reihenfolge (Gitterelektronengleichung - Einelektronengleichung - Kerngleichung) nacheinander zu lösen. Die Wahrung solcher Strenge wäre physikalisch jedoch unvernünftig, weil bei der Theorie des $F$-Zentrums nicht alle drei Untersysteme gleich interessant sind. So sind die Differenzierungen innerhalb des Gitterelektronensystems in diesem Rahmen ziemlich bedeutungslos, und wichtig ist nur die Gesamtwirkung auf das Störstellenelektron und die Kerngleichung. Man kann also, wie es durchaus der physikalischen Realität entspricht, Gitterelektronen und Kerne zu komplexen Einzelteilchen (Ionen) zusammenfassen,

\footnotetext{
2 S. I. PEKAr, Unters. üb. d. Elektronentheorie der Kristalle, Akademie-Verlag, Berlin 1954; dort weitere Literaturzitate. - F. C. Williams, J. Chem. Phys. 19, 457 [1951].
} 